\title{
Characterization of Families of Rank 3 Permutation Groups by the Subdegrees II $^{1}$ )
}

\author{
By
}

D. G. HIGMaN

1. Introduction. This paper is a continuation of an investigation of the extent to which the known families of rank 3 permutation groups are determined by their subdegrees, two families having been considered from this point of view in [4]. The main result to be proved here is the following theorem, in which

$$
Q_{N} \equiv Q_{N}(q)=q^{N-1}+q^{N-2}+\cdots+1 .
$$

Theorem III. Let $G$ be a rank 3 group of permutations of degree

with subdegrees $k=q Q_{2} Q_{N-2}$ and

$$
n=\frac{Q_{N} Q_{N-1}}{Q_{2}}
$$

$$
l=q^{4} \frac{Q_{N-2} Q_{N-3}}{Q_{2}}
$$

where $q \geqq 2$ and $N$ are integers. Then for all even values of $N \geqq 6$ and all odd values of $N \geqq 19, G$ is isomorphic with a subgroup $H$ of $P \Gamma L_{N}(q)$ regarded as a group of permutations of the set of lines of $(N-1)$-dimensional projective space $\mathbf{P}_{N-1}(q)$ over $\mathbf{F}_{q}$, such that $H$ is transitive on the set of 4-simplices in $\mathbf{P}_{N-1}(q)$.

The conditions on $N$ can be replaced by the assumption that $N$ is at least 6 and the intersection number $\mu$ has the value $(q+1)^{2}$. The cases $N=4$ and 5 are unsettled even under the assumption that $\mu$ has this value.

We number the main theorems of this sequences of papers consecutively, Theorems I and II having appeared in [4]. Theorem III can be regarded as a linear analogue of Theorem II, which in turn corresponds to the case $q=1$ of Theorem III. The subgroups of $P \Gamma L_{N}(q)$ transitive on the set of 4-simplices in $\mathbf{P}_{N-1}(q)$ can be regarded as linear analogues of 4-fold transitive permutation groups of degree $N$. It does not appear to be known whether $P S L_{N}(q)$ is always the minimal such group.

Since this paper was submitted, D. Perrir has proved that a subgroup of $P \Gamma L_{N}(q), q \neq 2$, which is transitive on the set of $k$-dimensional linear subvarieties of $\mathbf{P}_{N-1}(q)$ for some $k, 2 \leqq k \leqq[N / 2]-1$, contains $P S L_{N}(q)$. This implies in particular that a subgroup of $P \Gamma L_{N}(q), N \geqq 6, q \neq 2$, which has rank 3 on the set of lines

1) Research supported in part by the National Science Foundation. 
contains $P S L_{N}(q)$. The statement of Theorem IV can be ammended accordingly and the last part of the conclusion of Theorem III can be improved to read that $H$ contains $P S L_{N}(q)$ provided that $q \neq 2$.

It will be convenient to refer to section 2 of [4] for the basic facts needed concerning strongly regular graphs and rank 3 groups.

2. The graphs $\mathscr{L}_{N}(q)$. Let $V$ be a vector space of dimension $N \geqq 2$ over the field $\mathbf{F}_{q}$ of $q$ elements and let $\mathscr{S}_{k}=\mathscr{S}_{k}(V)$ be the set of all $k$-dimensional subspaces of $V$. We denote by $\mathbf{P}=\mathbf{P}(V)$ the lattice of all subspaces of $V$ regarded as an $(N-1)$ dimensional projective space over $\mathbf{F}_{q}$, so that $\mathscr{S}_{k}$ is the set of $(k-1)$-dimensional linear subspaces of $\mathbf{P}, \mathscr{S}_{1}, \mathscr{S}_{2}, \mathscr{S}_{3}$ and $\mathscr{S}_{4}$ being respectively the sets of points, lines, planes and 3-spaces. If we put $Q_{N}=Q_{N}(q)=q^{N-1}+q^{N-2}+\cdots+q+1$ and

$$
Q_{N, k}=\frac{Q_{N} Q_{N-1} \cdots Q_{N-k+1}}{Q_{k} Q_{k-1} \cdots Q_{1}}
$$

then $\left|\mathscr{S}_{k}\right|=Q_{N, k}$. We can include the case $q=1$ by letting $V$ be a set of $N$ elements and $\mathscr{S}_{k}$ the set of all $k$-element subsets of $V$ in this case. For $q \geqq 2$ and $N \geqq 3$ the automorphism group of $\mathbf{P}$ is the group $P \Gamma L(V)$ induced by the group $\Gamma L(V)$ of all nonsingular semilinear transformations of $V$, while for $q=1$ it is the group induced by the symmetric group on $V$.

For $N \geqq 4, P \Gamma L(V)$ acts faithfully as a rank 3 group of permutations of the set $\mathscr{S}_{2}$ of lines, with degree and subdegrees

$$
n=Q_{N, 2}, \quad k=q Q_{2} Q_{N-2} \quad \text { and } \quad l=q^{4} Q_{N-2,2}
$$

and intersection numbers

$$
\lambda=Q_{N-1}+q^{2}-2 \text { and } \mu=(q+1)^{2} .
$$

The same is true of the subgroup $P S L(V)$ induced by the special linear group $S L(V)$. This means that the graph having as vertex set the set $\mathscr{S}_{2}$ of lines, with two being adjacent if and only if they intersect, is a strongly regular graph with parameters $n, k, l, \lambda, \mu$ (in the notation of [4]) given by (1) and (2), admitting $P \Gamma L(V)$ and its subgroup $P S L(V)$ as rank 3 groups of automorphisms.

When we have fixed an $N$-dimensional vector space $V$ over $\mathbf{F}_{q}$, we often write $\mathbf{P}_{N-1}(q), P \Gamma L_{N}(q)$ and $\mathscr{L}_{N}(q)$ for $\mathbf{P}(V), P \Gamma L(V)$ and $\mathscr{L}(V)$ respectively.

3. Rank 3 graphs and the 4 -vertex condition. A strongly regular graph admitting a rank 3 group of automorphisms will be called a rank 3 graph; the graphs $\mathscr{L}_{N}(q)$, $N \geqq 4, q \geqq 1$ are rank 3 graphs.

The problem of determining the rank 3 permutation groups of even order with specified degree $n$ and subdegrees $k$ and $l$ breaks down into the following two problems:

1. Determination of the rank 3 graphs with the specified $n, k$ and $l$ as parameters (in the notation of [4]), and

2. Determination of the rank 3 subgroups of the automorphism groups of these graphs.

Concerning 1 . we remark that the problem of determining the strongly regular graphs with given parameters $n, k$ and $l$ is in general very much more difficult than 
that of determining the rank 3 graphs. For example, the line graph (in the terminology of SIMS [11], cf. section 4 of the present paper) of a Steiner triple system with $Q_{N}(2)$ vertices, $N \geqq 4$, is a strongly regular graph satisfying (1) and (2). The graphs $\mathscr{L}_{N}(2)$ are included, but there are many others (of. [10]).

It is natural to introduce classes of graphs between strongly regular graphs and rank 3 graphs. By a (t-vertex) subgraph of a graph $\mathscr{G}$ we mean a subset $S$ of the vertex set of $\mathscr{G}$ (with $|S|=t$ ) together with all edges in $\mathscr{G}$ joining vertices in $S$. If $X$ is a set of vertices in $\mathscr{G}$ and $\mathscr{G}_{1}$ and $\mathscr{G}_{2}$ are subgraphs of $\mathscr{G}$, we say that $\mathscr{G}_{1}$ and $\mathscr{G}_{2}$ are of the same type with respect to $X$ if $X$ is contained in the vertex set of $\mathscr{G}_{1}$ and of $\mathscr{G}_{2}$, and there is an isomorphism of $\mathscr{G}_{1}$ onto $\mathscr{G}_{2}$ fixing $X$ pointwise. A strongly regular graph will be said to satisfy the t-vertex condition if, for each twoelement subset $X$ of its vertex set, the number of $t$-vertex subgraphs of each type with respect to $X$ depends only on whether $X$ is an edge or not. A strongly regular graph satisfies the $t$-vertex condition for $t \leqq 3$ but need not satisfy the 4 -vertex condition. A rank 3 graph satisfies the $t$-vertex condition for all $t$.

If $G$ is a strongly regular graph satisfying the 4-vertex condition, then the parameters $\alpha$ and $\beta$ introduced by Srms [11] for rank 3 graphs, namely

$$
\alpha=\text { the number of 4-cliques containing a given edge, }
$$

and

$$
\beta=\begin{aligned}
& \text { the number of subgraphs of type } x<y \text { of nonadjacent vertices } \\
& x, y \text { with respect to a given pair }
\end{aligned}
$$

are defined independently of the given edge and nonadjacent pair of vertices respectively. The relation

$$
k\left\{\left(\begin{array}{l}
\lambda \\
2
\end{array}\right)-\alpha\right\}=l \beta
$$

given by Sms [11, Lemma 2.4] holds. The classification result for graphs given in this paper (Lemma 5) is for strongly regular graphs satisfying (1) and the 4-vertex condition.

4. The intersection numbers. Throughout this section we let $\mathscr{G}$ be a strongly regular graph with parameters $n, k$ and $l$ given by (1) for some integers $q \geqq 2$ and $N \geqq 4$ (the case $q=1$ having already been considered in [4]). Concerning the possible values for the intersection number $\mu$ we prove first that

Lemma 1. (i) If $N$ is even, then $\mu=(q+1)^{2}$.

(ii) If $N$ is odd, then $\mu=(q+1)^{2}+(q+1) \frac{\varrho}{a}$ where $a=\left(q+1, Q_{(N-3) / 2}\left(q^{2}\right)\right)$ and $\varrho$ is an integer such that $-a(q+1)<\varrho \leqq \frac{q+1}{q} a$.

Proof. If $\mu=0$, then $k+1 \mid n$ by $[4,(8)]$. But

$$
\begin{aligned}
k+1 & =q(q+1) Q_{N-2}+1=Q_{N}+Q_{N-1}-(q+1)= \\
& =Q_{N-1}+q^{2} Q_{N-2}=Q_{N}+q^{2} Q_{N-3} .
\end{aligned}
$$

Hence, on the one hand, $\left(k+1, Q_{N-1}\right)=1$, so that $k+1 \mid Q_{N}$, while on the other, $k+1>Q_{N}$, a contradiction. Hence $\mu>0$. 
By $[4,(3)]$,

(4)

$$
q^{3} Q_{N-3} \mu=Q_{2}^{2}\left(q Q_{2} Q_{N-2}-(\lambda+1)\right)
$$

giving

$$
\mu=Q_{2}^{2}\left\{1+\frac{Q_{N-4}-\gamma}{Q_{N-3}}\right\}
$$

where $\gamma$ is an integer such that $\lambda=\gamma q^{3}+2 q^{2}+q-1$. Since $\lambda \geqq 0, \gamma<0 \mathrm{im}$ plies that $\gamma=-1$ and $q=2$, so that

$$
\mu=9\left\{1+\frac{2^{N-4}}{2^{N-3}-1}\right\}
$$

and hence $N \leqq 5$. If $N=4$, then $\mu=k=18$, which is impossible by [4, (8)] since $l+1=17$ does not divide $n=35$. If $N=5$, then $\mu=15, \lambda=1$ and $k=42$, giving $d=304$, which is impossible by $[4,(6)]$. Hence $\gamma \geqq 0$.

If $N$ is even, $\left(Q_{2}, Q_{N-3}\right)=1$ so that $\gamma=Q_{N-4}-\varrho Q_{N-3}$ with $\varrho$ an integer $\leqq 0$. Since $\mu>0, \varrho \neq 0$ implies $\gamma<0$, a contradiction. This proves (i).

Assume now that $N$ is odd, so that $Q_{N-3}=(q+1) Q_{(N-3) / 2}\left(q^{2}\right)$. Then by (5),

$$
\mu=(q+1)^{2}+(q+1) \frac{\varrho}{a} \text { and } a \gamma=a Q_{N-4}-\varrho Q_{(N-3) / 2}\left(q^{2}\right)
$$

with $a=\left(q+1, Q_{(N-3) / 2}\left(q^{2}\right)\right)$ and $\varrho$ an integer. Since $\mu>0$ and $\gamma \geqq 0$ we must have $-a(q+1)<\varrho \leqq \frac{q+1}{q} a$.

The next lemma implies the existence of a bound on the values of $N$ for which $\mu \neq(q+1)^{2}$ is possible.

Lemma 2. Put $A=(q+1)^{3}-q \mu$ and

Then

$$
B=(q+1)^{2}\left(q^{2}+2 q-1\right)+\left(q^{2}-q-1\right) \mu .
$$

and

$$
q^{N-1}=\frac{A B-2(q-1)(q+1)^{5} \pm w}{A^{2}}
$$

(7) $w^{2}-A^{2} y^{2}=4(q-1)^{2}(q+1)^{4} q \mu\left(\mu-(q+1)^{2}\right)\left(q \mu-(q+1)\left(q^{2}+q+1\right)\right)$

for integers $w$ and $y$ (where $d=(q-1)^{2}(q+1)^{4} y^{2}$ in the notation of $[4,(6)]$ ).

Proof. By (4),

$$
(q+1)^{2} \lambda=(q+1)^{3} \frac{q^{N-1}-q}{q-1}-(q+1)^{2}-q \frac{q^{N-1}-q^{2}}{q-1} \mu .
$$

Putting $z=q^{N-1}$ we get

and

$$
(q+1)^{2}(\lambda-\mu)=\frac{1}{q-1}\{A z-B\}
$$

$$
k-\mu=\frac{1}{q-1}\{(q+1) z-C\}
$$

where $C=q(q+1)+(q-1) \mu$. By $[4,(6)]$ there is an integer $y>0$ such that

$$
\begin{aligned}
y^{2} & =(q-1)^{2}(q+1)^{4}\left\{(\lambda-\mu)^{2}+4(k-\mu)\right\}= \\
& =A^{2} z^{2}-2\left(A B-2(q-1)(q+1)^{5}\right) z+B^{2}-4(q-1)(q+1)^{4} C .
\end{aligned}
$$


Letting $2 w$ be the discriminant of this equation regarded as a quadratic in $z$, so that $w$ is an integer, we have equation (6) and

$$
\begin{gathered}
w^{2}-A^{2} y^{2}=A^{2} B^{2}-4(q-1)(q+1)^{5} A B+4(q-1)^{2}(q+1)^{10}- \\
\quad-A^{2}\left(B^{2}-4(q-1)(q+1)^{4} C\right)= \\
=4(q-1)(q+1)^{4} A\{A C-(q+1) B\}+4(q-1)^{2}(q+1)^{10}= \\
=4(q-1)^{2}(q+1)^{4} A\left\{-q \mu^{2}+q^{2}(q+1) \mu-(q+1)^{3}\right\}+ \\
+4(q-1)^{2}(q+1)^{10}
\end{gathered}
$$

which reduces to (7).

From Lemma 2 we see that

Lemma 3. $\mu=(q+1)^{2}$ for all (odd) values of $N \geqq 19$.

Proof. By Lemma $1,1 \leqq \mu \leqq(q+1)(q+2)$, so

and, by (7),

$$
q+1<A<(q+1)^{3}, \quad B<2(q+1)^{4}
$$

$$
w^{2}-A^{2} y^{2}=4 K, \quad|K|<(q-1)^{2}(q+1)^{12} .
$$

Assume $\mu \neq(q+1)^{2}$, then $K \neq 0$ so that $|w|<2(q-1)^{2}(q+1)^{12}$. Now (6) gives $\frac{1}{2} q^{N-1}<(q+1)^{5}+(q-1)^{2}(q+1)^{10}$, which is violated whenever $q \geqq 2$ and $N \geqq 19$.

5. Characterization of the graphs. In characterizing our graphs we follow the method of SIMS [11], making use of a result of BOSE [3]. For this we define a configuration to be a set $S$ of points and a set $\mathscr{L}$ of nonempty subsets of $S$, called lines, such that there is at most one line containing any two distinct points. The line graph of such a configuration $(S, \mathscr{L})$ has for its vertices the lines, two being adjacent if and only if they intersect. Thus $\mathscr{L}_{N}(q)$ is the line graph of the configuration of points and lines of $\mathbf{P}_{N-1}(q)$. We state the result of BOSE [3; Th. 9.3], dualized as indicated by Sims [11; Lemma 2.6], as

Lemma 4. Let $\mathscr{G}$ be a strongly regular graph with parameters $n, k, l, \lambda, \mu$ and minimum eigenvalue $s=-m$. Assume that $t=\mu / m$ is an integer, and that

1) $t \leqq m, \quad t \leqq \lambda-(m-1)(t-1)+2$

and

2) $\lambda-(m-1)(t-1)+2>\frac{1}{2}\left[m(m-1)+t(m+1)\left(m^{2}-2 m+2\right)\right]$.

Then $\mathscr{G}$ is isomorphic with the line graph of a configuration such that

each line carries exactly $m$ points, each point lies on exactly $\lambda-(m-1)(t-1)+2$

(8) lines, and through each point $P$ there pass exactly $t$ lines meeting any given line not through $P$ 2).

We use Lemma 4 and SIMs' method [11] to prove

Lemma 5. Let $\mathscr{G}$ be a strongly regular graph satisfying (1) for some integers $q \geqq 2$ and $N \geqq 6$. Assume that $\mu=(q+1)^{2}$ and that $\mathscr{G}$ satisfies the 4-vertex condition. Then $q$ is a prime power and $\mathscr{G} \approx \mathscr{L}_{N}(q)$.

2) A configuration satisfying (8) is a partial geometry in the terminology of [3]. 
Proof. By Lemma 4, $\mathscr{G}$ is the line graph of a configuration $(S, \mathscr{L})$ such that

(9) $|S|=Q_{N}$, each line carries $q+1$ points, each point lies on $Q_{N-1}$ lines, and two distinct points lie on exactly one line.

For, since $\mu=(q+1)^{2}, m=t=q+1$ and $\lambda=Q_{N-1}+q^{2}-2$. Condition 1) of Lemma 2 is satisfied and condition 2 ) becomes

$$
Q_{N-1}>\frac{1}{2}\left[(q+1) q+(q+1)(q+2)\left\{(q+1)^{2}-2(q+1)+2\right\}\right]
$$

which reduces to $2 q^{N-1}>q\left(q^{4}+q^{2}-2\right)$. This inequality is satisfied for $q \geqq 2$ and $N \geqq 6$.

To complete the proof of Lemma 5 we must show that $(S, \mathscr{L})$ is isomorphic with the configuration of points and lines of a projective space over $\mathbf{F}_{q}$. For this it suffices (cf. [1]) to show that any two transversals of a given pair of intersecting lines intersect.

The number of transversals of two intersecting lines $l$ and $m$ is $q^{2}$. The parameter $\alpha$, whose value is the number of 4 -cliques in $\mathscr{G}$ containing $l$ and $m$, has the form

$$
\alpha=\left(\begin{array}{c}
Q_{N-1}-2 \\
2
\end{array}\right)+f
$$

where $q^{2}(q-1) \leqq f \leqq q^{2}(q-1)+\left(\begin{array}{c}q^{2} \\ 2\end{array}\right)$, the upper bound being attained precisely when any two transversals intersect.

We have

and by (3),

$$
\left(\begin{array}{l}
\lambda \\
2
\end{array}\right)-\alpha=\frac{q^{2}}{2(q-1)}\left\{2 q^{N-1}+\left(q^{3}-q^{2}-5 q+3\right)\right\}-f
$$

$$
q(q+1)\left\{\left(\begin{array}{l}
\lambda \\
2
\end{array}\right)-\alpha\right\}=q^{4} \frac{q^{N-3}-1}{(q-1)(q+1)} \beta .
$$

Hence $q^{2} \mid 2 f, 2 f=q^{2} f_{0}, 2(q-1) \leqq f_{0} \leqq(q-1)(q+3)$, and

i.e.,

$$
(q+1)^{2}\left\{2 q^{N-1}+\left(q^{3}-q^{2}-5 q+3\right)-(q-1) f_{0}\right\}=2 q\left(q^{N-3}-1\right) \beta
$$

$$
(q+1)^{2}\left\{2 q^{2}\left(q^{N-3}-1\right)+(q-1)^{2}(q+3)-(q-1) f_{0}\right\}=2 q\left(q^{N-3}-1\right) \beta .
$$

Hence $\left.2 q\left(q^{N-3}-1\right)\right](q+1)^{2}(q-1)\left[(q-1)(q+3)-f_{0}\right]$, and so, if $f_{0}<$ $(q-1)(q+3)$, we have

$$
2 q\left(q^{N-3}-1\right) \leqq(q+1)^{2}(q-1)[(q-1)(q+3)-2(q-1)] \leqq(q+1)^{3}(q-1)^{2}
$$

This implies that $N \leqq 6$. Since $\left(q^{3}-1, q+1\right) \mid 2$, we have for $N=6$ that

$$
2 q\left(q^{3}-1\right) \leqq 4(q-1)^{2}(q+1), \text { i.e., } q\left(q^{2}+q+1\right) \leqq 2\left(q^{2}-1\right),
$$

which is impossible.

Lemma 4 cannot be applied in the cases $N=4$ and 5 , and we don't know if in these cases $\mathscr{G}$ as in Lemma 5 is the line graph of a configuration satisfying (9). If we assume that $\mathscr{G}$ is the line graph of a configuration satisfying (9) for some $q \geqq 2$ and $N \geqq 4$, then $\mathscr{G}$ is strongly regular and satisfies (1). If $\mathscr{G}$ satisfies the 4 -vertex condition, then for $N=4$ or 5 , our conditions on $f_{0}$ reduce to

$$
2 q \mid(q-1)(q+3)-f_{0} \text { and } 2(q-1) \leqq f_{0} \leqq(q-1)(q+3)
$$


At this point we do not know whether any of the exceptional values are realized by actual configurations or not. Conceivably some of the Steiner triple systems might give graphs satisfying the 4 -vertex condition with exceptional values of $\alpha$.

6. The rank 3 groups. We now examine the rank 3 automorphism groups of the line graph of the configuration of points and lines of a finite projective space. We begin with two simple lemmas relating the automorphism groups of configurations and their line graphs.

Let $(S, \mathscr{L})$ be a configuration, in the sense of section 5 , satisfying (8), and let $\mathscr{G}$ be its line graph. There is an injection of the automorphism group $H$ of $(S, \mathscr{L})$ into the automorphism group $G$ of $\mathscr{G}$ corresponding to the faithful action of $H$ on the set of lines of $(S, \mathscr{L})$; we regard $H$ as a subgroup of $G$.

Lemma 6. If $(S, \mathscr{L})$ is a configuration satisfying (8), and if

then $H=G$.

$$
\lambda>2(m-1)(t-1)+t-2,
$$

Proof. In $\mathscr{G}$ there are just two types of maximal cliques, namely

(I) those consisting of all lines through a given point, and

(II) those containing triangle of $(S, \mathscr{L})$.

The number of vertices in a clique of type (I) is $\lambda=(m-1)(t-1)+2$, while the number in a clique of type (II) is at most the number of transversals of a given pair $l, m$ of intersecting lines, plus the number of lines through $l \cap m$ meeting a given transversal, i.e., $(m-1)(t-1)+t$. Hence if the given inequality holds, then each element of $G$ induces a permutation of the points of $(S, \mathscr{L})$ and so belongs to $H$.

Lemma 7. Let $(S, \mathscr{L})$ be a configuration satisfying (9) with $q \geqq 2$ and $N \geqq 4$, and assume that the line graph $\mathscr{G}$ satisfies the 4-vertex condition. Then either (a) the automorphism group of $(S, \mathscr{L})$ coincides with that of $\mathscr{G}$, or (b) $N=4,(S, \mathscr{L})$ is the configuration of points and lines of a projective 3-space over $\mathbf{F}_{q}$, and the automorphism group of $(S, \mathscr{L})$ has index 2 in that of $\mathscr{G}$.

Proof. In this case the inequality of Lemma 6 becomes $Q_{N-1}>Q_{3}$ so that Lemma 6 applies for $N \geqq 5$. If $N=4$, the argument of the proof of Lemma 6 fails precisely if there exists a pair $l, m$ of intersecting lines for which every pair of transversals intersect. Since we are assuming the 4-vertex condition this means that every pair of intersecting lines has this property. Hence by the axioms given for projective geometry in [1], $(S, \mathscr{L})$ is the configuration of points and lines of a projective space of dimension 3 over $\mathbf{F}_{q}$, and it is easy to see that the full automorphism group of $\mathscr{G}$ is $\left\langle P \Gamma L_{4}(q), \delta\right\rangle$ where $\delta$ is a polarity.

We need the following linear analogue of the result [6; Th. I] of LIvingstone and WAGNER, which has been proved by McLadghtinN [8].

Lemma 8. If $1 \leqq k^{\prime} \leqq k \leqq N / 2$ and $G \leqq P \Gamma L_{N}(q)$, then the number of orbits for $G$ in $\mathscr{P}_{k^{\prime}}$ is not greater than the number in $\mathscr{S}_{k}$.

Using this result we prove 
Lemma 9. $A$ subgroup $G$ of $P \Gamma L_{N}(q), N \geqq 4$, has rank 3 on the set $\mathscr{I}_{2}$ of lines of $\mathbf{P}=\mathbf{P}_{N-1}(q)$ if and only if $G$ is transitive on the set $\mathscr{S}_{4}$ of 3-spaces in $\mathbf{P}$, and, for $S \in \mathscr{S}_{4}$, $G_{S} \mid S$ contains $P S L_{4}(S)$.

Proof. We assume that $G$ has rank 3 on $\mathscr{S}_{2}$ and prove that it has the stated properties with respect to $\mathscr{S}_{4}$, the reverse implication being immediate.

The assumption implies at once that $G$ is transitive on each of the sets $\mathscr{S}_{2}, \mathscr{S}_{3}$ and $\mathscr{S}_{4}$. For $\pi \in \mathscr{S}_{3}, G_{\pi}$ is doubly transitive on the set of lines of $\pi$, so $G$ is doubly transitive on the set $\mathscr{S}_{1}$. For $S \in \mathscr{S}_{4}, G_{S} \mid S$ is transitive on the set of lines of $S$, and hence by Lemma 8 , on the set of points of $S$.

Taking $P \in \mathscr{S}_{1}, l \in \mathscr{P}_{2}$ and $S \in \mathscr{S}_{4}$ such that $P \subseteq l \subseteq S$, we have the index diagram

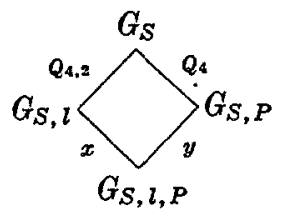

so that $Q_{3} x=Q_{2} y$. Since $\left(Q_{2}, Q_{3}\right)=1$ and $x \leqq Q_{2}$, it follows that $x=Q_{2}$ and $y=Q_{3}$. In particular, $G_{S, l}$ is transitive on the points of $l$.

Now let $Q$ be a point $\neq P$ on $l$. From the index diagram

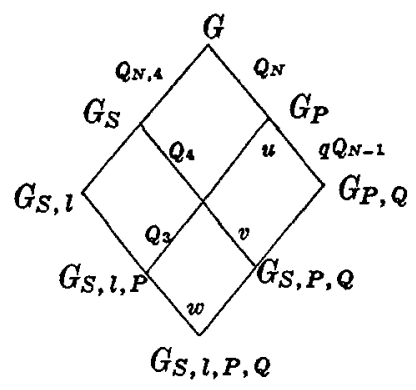

we see that $u$ is prime to $q$, so $q \mid v$ and hence $q \mid w$. This implies that $G_{S, l}$ is doubly transitive on the points of $l$.

We now have that $G_{S}$ is doubly transitive on the points of $S$ for $S \in \mathscr{P}_{4}$. Hence WAGNER's theorem [12], if $G_{S} \mid S$ does not contain $P S L_{4}(q)$ we must have $N=4$, $q=2$ and $G_{S} \mid S \approx A_{7}$. In this case there are 16 lines in $S$ not meeting a given line $l \subseteq S$. Hence 16 divides the order of $G_{S} \mid S$ since $G_{S, l}$ is transitive on these. But $16+\left|A_{7}\right|$.

From Lemmas 5 and 9 we have

Lemma 10. If $G$ is a rank 3 permutation group with degree and subdegrees given by (1) with $q \geqq 2$ and $N \geqq 6$, and if $\mu=(q+1)^{2}$, then the conclusion of Theorem $I I I$ holds.

Theorem III as stated in the introduction follows immediately from Lemmas 1 , 3 and 10. 
The description of the rank 3 automorphism groups of $\mathscr{L}_{N}(q)$ can be rounded out to

Theorem IV. Let $G$ be a rank 3 subgroup of the automorphism group of $\mathscr{L}_{N}(q), N \geqq 4$, $q \geqq 2$. If $N \geqq 5$, then $G$ is a subgroup of $P \Gamma L_{N}(q)$ transitive on the set of 4-simplices in $\mathbf{P}_{N-1}(q)$. If $N=4$, then $G \cap P \Gamma L_{N}(q)$ is a subgroup of $G$ of index $\leqq 2$, containing $P S L_{4}(q)$ unless $q=2$ and $G \cap P S L_{4}(2) \approx A_{7}$.

Proof. By Lemmas 7 and 9 we may assume that $N=4$ and $G \leqq P \Gamma L_{4}(q)$. By WAGNER's theorem [12] it suffices to prove that $G \cap P \Gamma L_{4}(q)$ is doubly transitive on the points. But this normal subgroup of $G$ is transitive on $\mathscr{S}_{2}$ since $G$ is primitive on $\mathscr{S}_{2}$. Hence by a result of McLaUGHLIN [8], it is doubly transitive on $\mathscr{S}_{1}$.

\section{References}

[1] G. BrRkhoff, Lattice Theory, 3rd ed. Providence 1967.

[2] D. M. BLoom, The subgroups of $P S L(3, q)$ for odd $q$. Trans. Amer. Math. Soc. 127, 150 -178 (1967).

[3] R. C. Bose, Strongly regular graphs, partial geometries and partially balanced designs. Pacific J. Math. 13, 389-419 (1963).

[4] D. G. HIgmar, Characterization of families of rank 3 permutation groups by the subdegrees. I. Arch. Math. 21, 151-156 (1970).

[5] D. G. HIGMar and J. E. McLaUGHLIN, Rank 3 subgroups of finite symplectic and unitary groups. J. Reine Angew. Math. 218, 174-189 (1965).

[6] D. Lrvingstone and A. WAGNER, Transitivity of finite permutation groups on unordered sets. Math. Z. 90, 393-403 (1965).

[7] J. E. McLaUGHIIN, Some groups generated by transvections. Arch. Math. 18, 364-368 (1967).

[8] J. E. McLaUghLIN, Oral communication.

[9] H. H. MrTCHeLL, Determination of the ordinary and modular ternary linear groups. Trans. Amer. Math. Soc. 12, 207-242 (1911).

[10] J. J. SEIDEL, Strongly regular graphs. Proc. Waterloo Conference on Combinatorics (to appear).

[11] C. C. Srms, On graphs with rank 3 automorphism groups. J. Comb. Theory (to appear).

[12] A. WAGNER, On collineation groups of finite projective spaces. Math. Z. 76, 411-426 (1961).

Eingegangen am 26. 8. 1969

Anschrift des Autors:

D. G. Higman

Department of Mathematics

University of Michigan

Ann Arbor, Mich. 48104, USA 